\title{
Robust Point-Based Feature Fingerprint Segmentation Algorithm
}

\author{
Chaohong Wu, Sergey Tulyakov, and Venu Govindaraju \\ Center for Unified Biometrics and Sensors (CUBS) \\ SUNY at Buffalo, USA
}

\begin{abstract}
A critical step in automatic fingerprint recognition is the accurate segmentation of fingerprint images. The objective of fingerprint segmentation is to decide which part of the images belongs to the foreground containing features for recognition and identification, and which part to the background with the noisy area around the boundary of the image. Unsupervised algorithms extract blockwise features. Supervised method usually first extracts point features like coherence, average gray level, variance and Gabor response, then a Fisher linear classifier is chosen for classification. This method provides accurate results, but its computational complexity is higher than most of unsupervised methods. This paper proposes using Harris corner point features to discriminate foreground and background. Shifting a window in any direction around the corner should give a large change in intensity. We observed that the strength of Harris point in the foreground area is much higher than that of Harris point in background area. The underlying mechanism for this segmentation method is that boundary ridge endings are inherently stronger Harris corner points. Some Harris points in noisy blobs might have higher strength, but it can be filtered as outliers using corresponding Gabor response. The experimental results proved the efficiency and accuracy of new method are markedly higher than those of previously described methods.
\end{abstract}

\section{Introduction}

The accurate segmentation of fingerprint images is key component to achieve high performance in automatic fingerprint recognition systems. If more background areas are included into segmented fingerprint of interest, more false features are possibly introduced into detected feature set; If some parts of foreground are excluded, useful feature points may be missed. There are two types of fingerprint segmentation algorithms: unsupervised and supervised. Unsupervised algorithms extract blockwise features such as local histogram of ridge orientation [12], gray-level variance, magnitude of the gradient in each image block [3], Gabor feature [45]. Practically, the presence of noise, low contrast area, and inconsistent contact of a fingertip with the sensor may result in loss of minutiae or more spurious minutiae. Supervised method usually first extracts several features like coherence, average gray level, variance and Gabor response [5]6]7], then a simple linear classifier is chosen for classification. This method provides accurate results, but its computational complexity is higher than most unsupervised methods.

Segmentation in low quality images faces several challenging technical problems. First problem is the presence of noise caused by dust and grease on the surface of livescan fingerprint scanners. Second problem is ghost images of fingerprints remaining 
from the previous image acquisition [7]. Third problem is low contrast fingerprint ridges generated through inconsistent contact press or dry/wet finger surface. Fourth problem is indistinct boundary if the features in the fixed size of window are used. Final problem is segmentation features being sensitive to the quality of image.

This paper proposes using Harris corner point features [89] to discriminate foreground and background. The Harris corner detector was developed originally as features for motion tracking, it can reduce significantly amount of computation compared to tracking every pixel. It is translation and rotation invariant but not scale invariant. We found that the strength of a Harris point in the foreground area is much higher than that of a Harris point in the background area. Some Harris points in noisy blobs might have higher strength, but it can be filtered as outliers using corresponding Gabor response. The experimental results proved the efficiency and accuracy of new method are much better than those of previously described methods. Furthermore, this segmentation algorithm can detect accurate boundary of fingerprint ridge regions, which is very useful in removing spurious boundary minutiae, and most current segmentation methods can not provide consistent boundary minutiae filtering.

\section{Features for Fingerprint Segmentation}

Feature selection is the first step for designing fingerprint segmentation algorithm. There are two general types of features used for fingerprint segmentation, i.e., block features and pointwise features. In [510] selected point features include local mean, local variance or standard deviation, and Gabor response of the fingerprint image. Local mean is calculated as Mean $=\sum_{w} I$, local variance is calculated as $V a r=$ $\sum_{w}(I-M e a n)^{2}, w$ is window size centered the processed pixel. The Gabor response is the smoothed sum of Gabor energies for eight Gabor filter responses. Usually the Gabor response is higher in the foreground region than that in the background region. The coherence feature indicates how strong the local window gradients centered the processed point in the same dominant orientation. Usually the coherence will be much higher in the foreground than in the background, but it may be influenced significantly by boundary signal and noisy signal. Therefore, single coherence feature is not sufficient for robust segmentation. Systematic combination of those features is necessary.

$$
\text { Coh }=\frac{\left|\sum_{w}\left(G_{s, x}, G_{s, y}\right)\right|}{\left|\sum_{w}\left(G_{s, x}, G_{s, y}\right)\right|}=\frac{\sqrt{\left(G_{x x}-G_{y y}\right)^{2}+4 G_{x y}^{2}}}{G_{x x}+G_{y y}}
$$

Because pointwise-based segmentation method is time consuming, blockwise features are usually used in the commercial automatic fingerprint recognition systems. Block mean, block standard deviation, block gradient histogram [12], block average magnitude of the gradient [11] are most common block features for fingerprint segmentation. In [12] gray-level pixel intensity-derived feature called block clusters degree $(\mathrm{CluD})$ is introduced. $C l u D$ measures how well the ridge pixels are clustering.

$$
E(x, y)=\log \left\{\int_{r} \int_{\theta}|F(r, \theta)|^{2}\right\}
$$




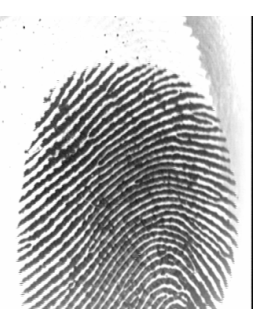

(a)

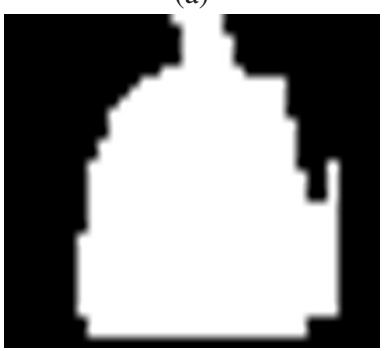

(c)

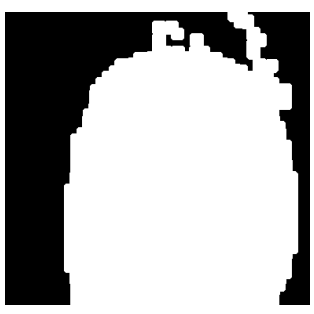

(e)

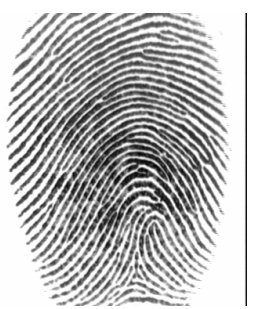

(b)

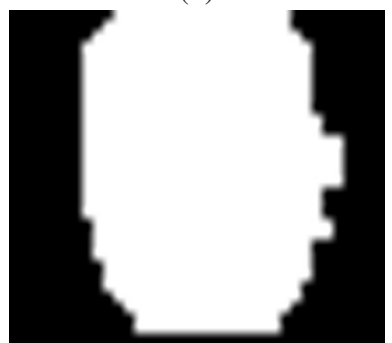

(d)
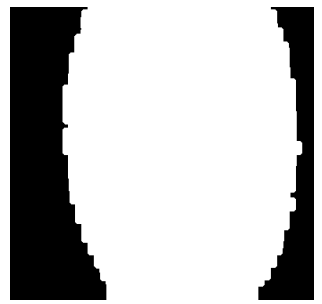

(f)

Fig. 1. (a) and (b) are two original images, (c) and (d) are FFT energy maps for images (a) and (b), (e) and (f) are Gabor energy maps for images (a) and (b), respectively

Texture features, such as Fourier spectrum energy [6], Gabor features [4]13] and Gaussian-Hermite Moments [14], have been applied to fingerprint segmentation. Ridges and valleys in a fingerprint image are generally observed to possess a sinusoidal-shaped plane wave with a well-defined frequency and orientation [15], and non-ridge regions does not hold this surface wave model. In the areas of background and noisy regions, it is assumed that there is very little structure and hence very little energy content in the Fourier spectrum. Each value of energy image E(x,y) indicates the energy content of the corresponding block. The fingerprint region may be differentiated from the background by thresholding the energy image. The logarithm values of the energy is used to convert the large dynamic range to a linear scale(Equation 2). The region mask is obtained by thresholding $E(x, y)$. However, uncleaned trace finger ridges and straight stripes are unfortunately included into regions of interest (Figure1 1 c)). 
Gabor filter-based segmentation algorithm is now most often used method [4[13]. An even symmetric Gabor filter has the following spatial form:

$$
g\left(x, y, \theta, f, \sigma_{x}, \sigma_{y}\right)=\exp \left\{-\frac{1}{2}\left[\frac{x_{\theta}^{2}}{\sigma_{x}^{2}}+\frac{y_{\theta}^{2}}{\sigma_{y}^{2}}\right]\right\} \cos \left(2 \pi f x_{\theta}\right)
$$

For each block of size $W \times W$ centered at (x,y), 8 directional Gabor features are computed for each block, the standard deviation of 8 Gabor features is utilized for segmentation. The formula for calculating the magnitude of Gabor feature is defined as,

$$
G\left(X, Y, \theta, f, \sigma_{x}, \sigma_{y}\right)=\left|\sum_{x_{0}=-w / 2}^{(w / 2)-1} \sum_{y_{0}=-w / 2}^{(w / 2)-1} I\left(X+x_{0}, Y+y_{0}\right) g\left(x_{0}, y_{0}, \theta, f, \sigma_{x}, \sigma_{y}\right)\right|
$$

However, fingerprint images with low contrast or false traces ridges or noisy complex background can not be segmented correctly by Gabor filter-based method (Figure1(e)).

In [14], similarity is found between Hermite moments and Gabor filter. GaussianHermite Moments has been successfully used to segment fingerprint images in [14]. Orthogonal moments use orthogonal polynomials as transform kernels and produce minimal information redundancy, Gaussian Hermite moments(GHM) can represent local texture feature without minimal noise effect.

\section{Harris Corner Points}

\subsection{Review of Harris-Corner-Points}

We propose using Harris corner point features [8[9] to discriminate foreground and background. The Harris corner detector was developed originally as features for motion tracking, it can reduce significantly amount of computation compared to tracking every pixel. Shifting a window in any direction around the corner should give a large change in intensity. Corner points provide repeatable points for matching, so some efficient methods have been designed [8]9]. Gradient is ill defined at a corner, so edge detectors perform poorly at corners. However, in the region around a corner, gradient has two or more different values. The corner point can be easily recognized by looking a small window. Shifting a window around a corner point in any direction should give a large change in gray-level intensity,

Given a point $\mathrm{I}(\mathrm{x}, \mathrm{y})$, and a $\operatorname{shift}(\Delta x, \Delta y)$, the auto-correlation function $\mathrm{E}$ is defined as:

$$
E(x, y)=\sum_{w(x, y)}\left[I\left(x_{i}, y_{i}\right)-I\left(x_{i}+\Delta x, y_{i}+\Delta y\right)\right]^{2}
$$

where $\mathrm{w}(\mathrm{x}, \mathrm{y})$ is window function centered on image point $(\mathrm{x}, \mathrm{y})$. For a small shifts $[\Delta x, \Delta y]$, the shifted image is approximated by a Taylor expansion truncated to the first order terms,

$$
I\left(x_{i}+\Delta x, y_{i}+\Delta y\right) \approx I\left(x_{i}, y_{i}\right)+\left[I_{x}\left(x_{i}, y_{i}\right) I_{y}\left(x_{i}, y_{i}\right)\right]\left[\begin{array}{c}
\Delta x \\
\Delta y
\end{array}\right]
$$


where $I_{x}\left(x_{i}, y_{i}\right)$ and $I_{y}\left(x_{i}, y_{i}\right)$ denote the partial derivatives in $\mathrm{x}$ and $\mathrm{y}$, respectively. Substituting approximation Equation 6 into Equation 5 yields,

$$
\begin{aligned}
E(x, y) & =\sum_{w(x, y)}\left[I\left(x_{i}, y_{i}\right)-I\left(x_{i}+\Delta x, y_{i}+\Delta y\right)\right]^{2} \\
& =\sum_{w(x, y)}\left(I\left(x_{i}, y_{i}\right)-I\left(x_{i}, y_{i}\right)-\left[I_{x}\left(x_{i}, y_{i}\right) I_{y}\left(x_{i}, y_{i}\right)\right]\left[\begin{array}{c}
\Delta x \\
\Delta y
\end{array}\right]\right)^{2} \\
& =\sum_{w(x, y)}\left(-\left[I_{x}\left(x_{i}, y_{i}\right) I_{y}\left(x_{i}, y_{i}\right)\right]\left[\begin{array}{c}
\Delta x \\
\Delta y
\end{array}\right]\right)^{2} \\
& =\sum_{w(x, y)}\left(\left[I_{x}\left(x_{i}, y_{i}\right) I_{y}\left(x_{i}, y_{i}\right)\right]\left[\begin{array}{c}
\Delta x \\
\Delta y
\end{array}\right]\right)^{2} \\
& =[\Delta x, \Delta y]\left[\sum_{w}\left(I_{x}\left(x_{i}, y_{i}\right)\right)^{2} \sum_{w} I_{x}\left(x_{i}, y_{i}\right) I_{y}\left(x_{i}, y_{i}\right)\right]\left[\begin{array}{c}
\Delta x \\
\Delta y
\end{array}\right] \\
& =[\Delta x, \Delta y] M(x, y)\left[\begin{array}{c}
\Delta x \\
\Delta y
\end{array}\right]
\end{aligned}
$$

That is,

$$
E(\Delta x, \Delta y)=[\Delta x, \Delta y] M(x, y)\left[\begin{array}{c}
\Delta a \\
\Delta y
\end{array}\right]
$$

where $\mathrm{M}(\mathrm{x}, \mathrm{y})$ is a $2 \times 2$ matrix computed from image derivatives, called auto-correlation matrix which captures the intensity structure of the local neighborhood.

$$
M=\sum_{x, y} w(x, y)\left[\begin{array}{cc}
\left(I_{x}\left(x_{i}, y_{i}\right)\right)^{2} & I_{x}\left(x_{i}, y_{i}\right) I_{y}\left(x_{i}, y_{i}\right) \\
I_{x}\left(x_{i}, y_{i}\right) I_{y}\left(x_{i}, y_{i}\right) & \left(I_{y}\left(x_{i}, y_{i}\right)\right)^{2}
\end{array}\right]
$$

\subsection{Strength of Harris-Corner Points of a Fingerprint Image}

In order to detect interest points, the original measure of corner response in [8] is :

$$
R=\frac{\operatorname{det}(M)}{\operatorname{Trace}(M)}=\frac{\lambda_{1} \lambda_{2}}{\lambda_{1}+\lambda_{2}}
$$

The auto-correlation matrix $(\mathrm{M})$ captures the structure of the local neighborhood. Based on eigenvalues $\left(\lambda_{1}, \lambda_{2}\right)$ of $\mathrm{M}$, interest points are located where there are two strong eigen values and the corner strength is a local maximum in a $3 \times 3$ neighborhood. To avoid the explicit eigenvalue decomposition of $\mathrm{M}, \operatorname{Trace}(M)$ is calculated as $I_{x}^{2}+I_{y}^{2}$, Det $(m)$ is calculated as $I_{x}^{2} I_{y}^{2}-\left(I_{x} I_{y}\right)^{2}$, and

$$
R=\operatorname{Det}(m)-k \times \operatorname{Trace}(M)^{2}
$$

To segment the fingerprint area (foreground) from the background, the following "corner strength" measure is used, because there is one undecided parameter $k$ in equa$\operatorname{tion}(11)$.

$$
R=\frac{I_{x}^{2} I_{y}^{2}-I_{x y}^{2}}{I_{x}^{2}+I_{y}^{2}}
$$




\subsection{Harris-Corner-Points Based Fingerprint Image Segmentation}

We found that the strength of a Harris point in the fingerprint area is much higher than that of a Harris point in background area, because boundary ridge endings inherently possess higher corner strength. Most high quality fingerprint images can be easily segmented by choosing appropriate threshold value. In Figure 2 a corner strength of 300 is selected to distinguish corner points in the foreground from those in the background. Convex hull algorithm is used to connect harris corner points located in the foreground boundary.

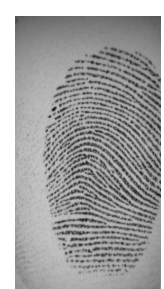

(a)

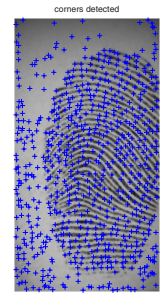

(b)

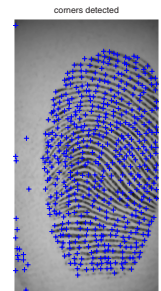

(c)

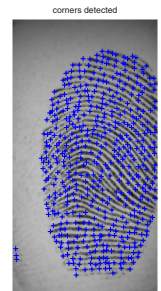

(d)

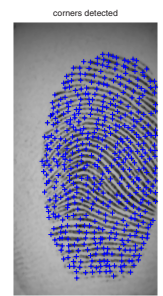

(e)

Fig. 2. A fingerprint with harris corner strength of (b)10, (c)60, (d)200, and (e)300. This fingerprint can be successfully segmented using corner response threshold of 300 .

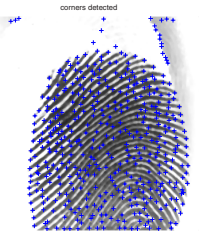

(a)

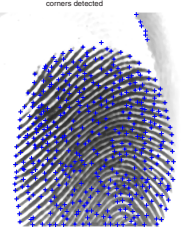

(b)

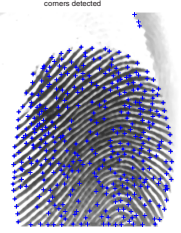

(c)

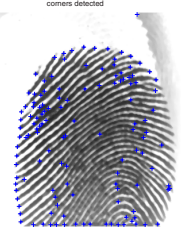

(d)

Fig. 3. A fingerprint with harris corner strength of (a)100, (b)500, (c)1000, (d) 1500 and (e)3000. Some noisy corner points can not be filtered completely even using corner response threshold of 3000 .

It appears relatively easy for us to segment fingerprint images for following image enhancement, feature detection and matching. However, two technical problems need to be solved. First, different "corner strength" thresholds are necessary to achieve good segmentation results for different qualities images based on image characteristical analysis. Second, Some Harris points in noisy blobs might have higher strength, it can not be segmented by choosing simply one threshold. When single threshold is applied to all the fingerprint images in one whole database, not all the corner points in the background in a fingerprint image are removed, some corner points in noisy regions can not be thresholded even using high threshold value (Figure 3). In order to deal with such situations, we implemented a heuristic selection algorithm using corresponding Gabor response (Figure 4 ). 


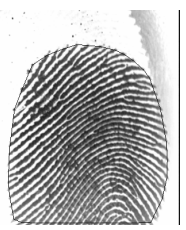

(a)

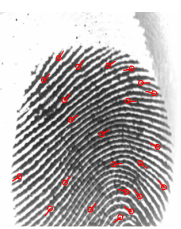

(b)

Fig. 4. Segmentation result and final feature detection result for the image shown in the Figure 1 a). (a) Segmented fingerprint marked with boundary line, (b) final detected minutiae.

\section{Experimental Results}

The proposed methodology is tested on FVC2002 DB1 and DB4, each database consists of 800 fingerprint images (100 distinct fingers, 8 impressions each). Image size is $374 \times$ 388 and the resolution is 500dpi. To evaluate the methodology of adapting a gaussian kernel to the local ridge curvature of a fingerprint image, we modified Gabor-based fingerprint enhancement algorithm [15 16] with two kernel sizes: the smaller one in high-curvature regions and the larger one in pseudo-parallel ridge regions, minutiae are detected using chaincode-based contour tracing [17], the fingerprint matcher developed by Jea et al. [18] is used for performance evaluation.

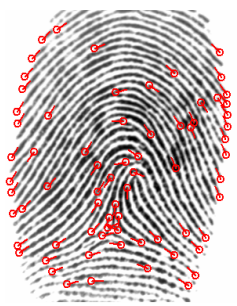

(a)

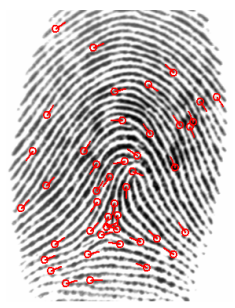

(c)

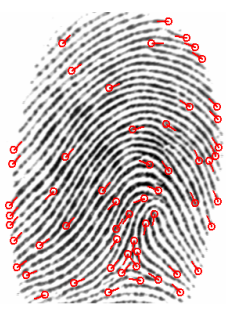

(b)

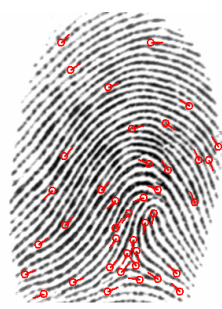

(d)

Fig. 5. Boundary spurious minutiae filtering. (a) and (b) incomplete filtering using NIST method, (c) and (d) proposed boundary filtering. 


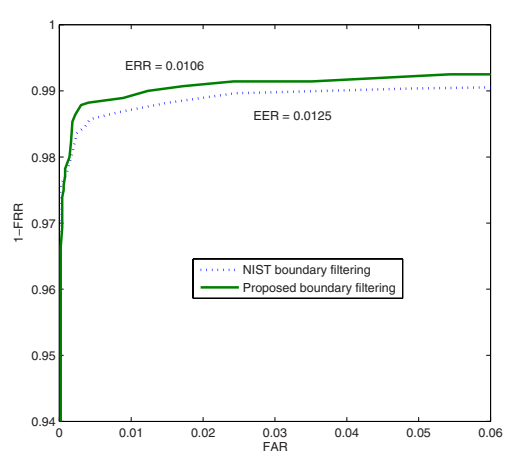

(a)

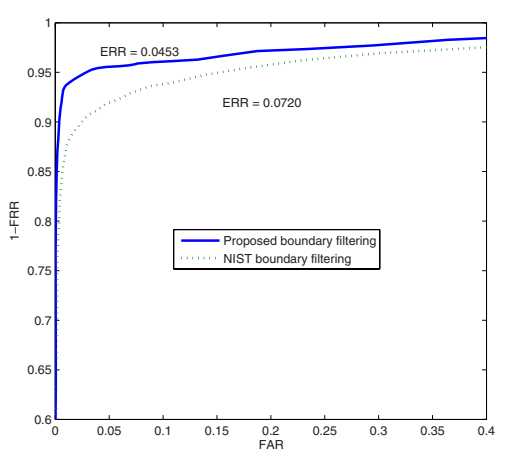

(b)

Fig. 6. ROC curves for (a) FVC2002 DB1 and (b) FVC2002 DB4

Our methodology has been tested on low quality images from FVC2002. To validate the efficiency of proposed segmentation method, current widely-used Gabor filterbased segmentation algorithm [4]13] and NIST segmentation [19] are utilized for comparison.

The proposed segmentation method have a remarkable advantage over current methods in terms of boundary spurious minutiae filtering. Figure 5 (a) and (b) show unsuccessful boundary minutiae filtering using NIST method [19], which is implemented by removing spurious minutiae pointing to invalid block and removing spurious minutiae near invalid blocks, and invalid blocks are defined as blocks with no detectable ridge flow. However, boundary blocks are more complicated, so the method in [19] fails to remove most boundary minutiae. In Figure 5 (c) and (d) show the filtering results of proposed method. In comparison of Figure 5 a) against (c) and (b) and (d), 30 and 17 boundary minutiae are filtered, respectively. Performance evaluations for FVC2002 DB1 and DB4 are shown in Figure 6 For DB1, ERR for false boundary minutiae filtering using proposed segmented mask is 0.0106 and EER for NIST boundary Filtering is 0.0125. For DB4, ERR for false boundary minutiae filtering using proposed segmented mask is 0.0453 and EER for NIST boundary Filtering is 0.0720 .

\section{Conclusions}

In this paper, a robust interest point based fingerprint segmentation is proposed for fingerprints of varied image qualities. The experimental results compared with those of previous methods validate that our algorithm has better performance even for low quality images, in terms of including less background and excluding less foreground. In addition, this robust segmentation algorithm is capable of filtering efficiently spurious boundary minutiae. 


\section{References}

1. Mehtre, B.M., Chatterjee, B.: Segmentation of fingerprint images - a composite method. Pattern Recognition 22(4), 381-385 (1989)

2. Mehtre, B.M., Murthy, N.N., Kapoor, S., Chatterjee, B.: Segmentation of fingerprint images using the directional image. Pattern Recognition 20(4), 429-435 (1987)

3. Ratha, N.K., Chen, S., Jain, A.K.: Adaptive flow orientation-based feature extraction in fingerprint images. Pattern Recognition 28(11), 1657-1672 (1995)

4. Alonso-Fernandez, F., Fierrez-Aguilar, J., Ortega-Garcia, J.: An enhanced gabor filter-based segmentation algorithm for fingerprint recognition systems. In: Pan, Y., Chen, D.-X., Guo, M., Cao, J., Dongarra, J.J. (eds.) ISPA 2005. LNCS, vol. 3758, pp. 239-244. Springer, Heidelberg (2005)

5. Bazen, A., Gerez, S.: Segmentation of fingerprint images. In: Proc. Workshop on Circuits Systems and Signal Processing (ProRISC 2001), pp. 276-280 (2001)

6. Pais Barreto Marques, A.C., Gay Thome, A.C.: A neural network fingerprint segmentation method. In: Fifth International Conference on Hybrid Intelligent Systems(HIS 05), p. $6(2005)$

7. Zhu, E., Yin, J., Hu, C., Zhang, G.: A systematic method for fingerprint ridge orientation estimation and image segmentation. Pattern Recognition 39(8), 1452-1472 (2006)

8. Harris, C., Stephens, M.: A combined corner and edge detector. In: Proc. in Alvey Vision Conference, pp. 147-151 (1988)

9. Mikolajczyk, K., Schmid, C.: Scale affine invariant interest point detectors. International Journal of Computer Vision 60(1), 63-86 (2004)

10. Klein, S., Bazen, A.M., Veldhuis, R.: fingerprint image segmentation based on hidden markov models. In: 13th Annual workshop in Circuits, Systems and Signal Processing, in Proc. ProRISC 2002 (2002)

11. Maio, D., Maltoni, D.: Direct gray-scale minutiae detection in fingerprints. IEEE Transactions on Pattern Analysis and Machine Intelligence 19(1), 27-40 (1997)

12. Chen, X., Tian, J., Cheng, J., Yang, X.: Segmentation of fingerprint images using linear classifier. EURASIP Journal on Applied Signal Processing 2004(4), 480-494 (2004)

13. Shen, L., Kot, A., Koo, W.: Quality measures of fingerprint images. In: Proc. Int. Conf. on Audio- and Video-Based Biometric Person Authentication, pp. 266-271 (2001)

14. Wang, L., Suo, H., Dai, M.: Fingerprint image segmentation based on gaussian-hermite moments. In: Li, X., Wang, S., Dong, Z.Y. (eds.) ADMA 2005. LNCS (LNAI), vol. 3584, pp. 446-454. Springer, Heidelberg (2005)

15. Hong, L., Wan, Y., Jain, A.K.: "Fingerprint image enhancement: Algorithms and performance evaluation". IEEE Transactions on Pattern Analysis and Machine Intelligence 20(8), 777-789 (1998)

16. $\mathrm{Wu}, \mathrm{C} .$, Govindaraju, V.: Singularity preserving fingerprint image adaptive filtering. In: International Conference on Image Processing, pp. 313-316 (2006)

17. Wu, C., Shi, Z., Govindaraju, V.: Fingerprint image enhancement method using directional median filter. In: Biometric Technology for Human Identification. SPIE, vol. 5404, pp. 66-75 (2004)

18. Jea, T.Y., Chavan, V.S., Govindaraju, V., Schneider, J.K.: Security and matching of partial fingerprint recognition systems. In: SPIE Defense and Security Symposium. SPIE, vol. 5404 (2004)

19. Watson, C.I., Garris, M.D., Tabassi, E., Wilson, C.L., MsCabe, R.M., Janet, S.: User's Guide to NIST Fingerprint Image Software2(NFIS2). NIST (2004)

20. Otsu, N.: A threshold selection method from gray level histograms. IEEE Transactions on Systems, Man and Cybernetics 9, 62-66 (1979) 